\title{
Effect of specialist palliative care services on quality of life in adults with advanced incurable illness in hospital, hospice, or community settings: systematic review and meta-analysis
}

\author{
Jan Gaertner, 1,2 Waldemar Siemens, ${ }^{1}$ Joerg J Meerpohl,,3,4 Gerd Antes, ${ }^{3}$ Cornelia Meffert, ${ }^{1}$ \\ Carola Xander, ${ }^{1}$ Stephanie Stock, ${ }^{5}$ Dirk Mueller, ${ }^{5}$ Guido Schwarzer, ${ }^{6}$ Gerhild Becker ${ }^{1}$
}

${ }^{1}$ Clinic for Palliative Care,

Medical Centre, University of

Freiburg, Faculty of Medicine,

University of Freiburg, Germany

${ }^{2}$ Palliative Care Centre

Hildegard, Basel, Switzerland

${ }^{3}$ Cochrane Germany, Medical

Centre, University of Freiburg,

Faculty of Medicine, University

of Freiburg, Germany

${ }^{4}$ Centre de Recherche

Épidémiologie et Statistique

Sorbonne Paris Cité-U1153,

Inserm/Université Paris

Descartes, Cochrane France,

Hôpital Hôtel-Dieu, 1 Place du

Parvis Notre Dame, 75181 Paris

Cedex 04, France

Institute for Health Economics and Clinical Epidemiology, Cologne University Hospital,

Cologne, Germany

${ }^{6}$ Institute for Medical Biometry and Statistics, Faculty of Medicine and Medical Centre,

University of Freiburg, Germany

Correspondence to: J Gaertner jan.gaertner@pzhi.ch

Additional material is published online only. To view please visit the journal online.

Cite this as: BMJ 2017;358:j2925 http://dx.doi.org/10.1136/bmj.j2925

Accepted: 5 June 2017

\section{ABSTRACT}

OBJECTIVE

To assess the effect of specialist palliative care on quality of life and additional outcomes relevant to patients in those with advanced illness.

DESIGN

Systematic review with meta-analysis.

\section{DATA SOURCES}

Medline, Embase, Cochrane Central Register of Controlled Trials, PsycINFO, and trial registers searched up to July 2016.

\section{ELIGIBILITY CRITERIA FOR SELECTING STUDIES}

Randomised controlled trials with adult inpatients or outpatients treated in hospital, hospice, or community settings with any advanced illness. Minimum requirements for specialist palliative care included the multiprofessional team approach. Two reviewers independently screened and extracted data, assessed the risk of bias (Cochrane risk of bias tool), and evaluated the quality of evidence (GRADE tool).

\section{DATA SYNTHESIS}

Primary outcome was quality of life with Hedges' $g$ as standardised mean difference (SMD) and random effects model in meta-analysis. In addition, the pooled SMDs of the analyses of quality of life were re-expressed on the global health/QoL scale (item 29 and 30, respectively) of the European Organization for Research and Treatment of Cancer QLQ-C30 (0-100, high values $=$ good quality of life, minimal clinically important difference 8.1).

\section{RESULTS}

Of 3967 publications, 12 were included (10 randomised controlled trials with 2454 patients randomised, of whom $72 \%$ ( $\mathrm{n}=1766$ ) had cancer). In no trial was integration of specialist palliative care triggered according to patients' needs as identified by screening. Overall, there was a small effect in favour of specialist palliative care (SMD 0.16, 95\% confidence interval 0.01 to 0.31; QLQ-C30 global health/QoL 4.1, 0.3 to $8.2 ; n=1218$, six trials). Sensitivity analysis showed an SMD of 0.57 ( -0.02 to 1.15; global health/ QoL 14.6, -0.5 to 29.4; $n=1385$, seven trials). The effect was marginally larger for patients with cancer $(0.20$, 0.01 to 0.38 ; global health/QoL 5.1, 0.3 to $9.7 ; n=828$, five trials) and especially for those who received specialist palliative care early $(0.33,0.05$ to 0.61 , global health/QoL 8.5, 1.3 to 15.6; $n=388$, two trials). The results for pain and other secondary outcomes were inconclusive. Some methodological problems (such as lack of blinding) reduced the strength of the evidence.

\section{CONCLUSIONS}

Specialist palliative care was associated with a small effect on QoL and might have most pronounced effects for patients with cancer who received such care early. It could be most effective if it is provided early and if it identifies though screening those patients with unmet needs.

SYSTEMATIC REVIEW REGISTRATION PROSPERO CRD42015020674.

\section{Introduction}

Palliative care is usually provided by physicians and other healthcare professional from all disciplines (such as family medicine, cardiology, oncology). This is known as "general palliative care" and forms the basis of excellent palliative care for most patients. ${ }^{1}$ In addition, specialist palliative care (specialised or specialty palliative care) has grown substantially. ${ }^{2}$ Moreover, the importance of meaningful, effective, and sustainable models of specialist palliative care is widely recognised. For example, the invited "perspective" on the development of such care published by Quill and Abernethy in 2013 has been cited by over 80 PubMed listed publications. ${ }^{1}$ The World Health Organization (WHO), the European Association of Palliative Care (EAPC), and other institutions strongly recommend the provision of specialist palliative care, ${ }^{34}$ but these recommendations are based on the descriptive analyses of available studies. ${ }^{5-7}$

We reviewed randomised controlled trials that compared the effect of specialist palliative care versus 
standard care on the quality of life (QoL; primary outcome), pain, and other outcomes in patients with advanced illness. Here, QoL is understood as health related QoL-that is, the patients' QoL with its physical, psychological and social dimensions as affected by the disease. ${ }^{89}$ This concept includes all domains of human suffering as in the physical, psychological, social, and spiritual domain. The main aim of palliative care is to improve QoL by preventing or relieving suffering in all of these dimensions. ${ }^{4}$ With our secondary outcomes we tried to capture various aspects of QoL (such as pain, dyspnoea, depression, anxiety, spiritual wellbeing, satisfaction with care, place of death, social wellbeing, and others).

\section{Methods}

This systematic review was registered ${ }^{10}$ (www.crd.york. ac.uk/PROSPERO/display_record.asp? ID= CRD42015020674), conducted according to Cochrane standards in cooperation with the German Cochrane Centre, and is reported in compliance with the PRISMA (preferred reporting items for systematic reviews and meta-analyses) statement. ${ }^{11}$ Part of the specific methods for specialist palliative care (for example, definition of the intervention, patients, and outcomes) was developed by a consensus project of two international working groups. ${ }^{12}$

\section{Inclusion criteria}

We included randomised controlled trials and cluster randomised controlled trials of specialist palliative care compared with standard care (full text articles and abstracts, no date or language restrictions) with adult (age $\geq 18$ ) inpatients and outpatients with advanced illness. We defined advanced illness as malignant and non-malignant diseases that lead to a decline in general health and eventually to death. ${ }^{13}$

Zimmermann and colleagues defined specialist palliative care as a "service of health care professionals from at least two different professions that provides or coordinates comprehensive care for patients."7 As palliative care aims to improve quality of life, we did not include study interventions focusing primarily on only one specific aspect (for example, symptoms like dyspnoea).

Studies that assessed specialist palliative care in hospitals, hospices, or community settings were eligible as well as studies with a minority $(<25 \%)$ of patients treated at home.

\section{Outcomes}

Our primary outcome was patients' quality of life. We included all tools that covered at least two dimensions of quality of life (physical, psychological or social). Secondary outcomes included symptom burden (pain, fatigue, nausea, and dyspnoea), psychosocial variables (distress, depression, anxiety, spiritual wellbeing, social wellbeing, and satisfaction), survival time, place of death, cost of care, and attrition (or completion rate). ${ }^{10}$

\section{Search}

We first searched Medline (via Ovid), Embase (via DIMDI), Cochrane Central Register of Controlled Trials
(CENTRAL, via Wiley), and PsycINFO (via EBSCO) in October 2015 (table A in appendix) and updated the search in July 2016. We used the Cochrane sensitivity and precision maximising search strategy ${ }^{14}$ and parts of the $B M J$ search filter ${ }^{15}$ to identify randomised controlled trials. We also searched palliative care textbooks, ${ }^{16-18}$ reference lists of relevant reviews, abstract books, and trial registers (www.controlled-trials.com, www. clinicaltrials.gov, apps.who.int/trialsearch/). Thirteen leading authors of relevant research were contacted to identify unpublished data.

\section{Data collection}

Two reviewers (JG, WS) independently screened titles and abstracts, and disagreements were resolved by discussion with a third reviewer (GB). Relevant data from the included studies were extracted mostly from the text or tables (WS, JG). If information was present only in figures, we planned to contact authors or extract the data with help of a large printout and the use of a ruler to measure and subsequently calculate relevant values. Risk of bias was assessed with the Cochrane Collaboration's risk of bias tool' ${ }^{19}$ (WS, JG). We used the GRADE (Grading of Recommendations Assessment, Development, and Evaluation) system to evaluate the quality of evidence (classification in high, moderate, low, very low). ${ }^{2021}$

\section{Data analysis}

RevMan 5.3 was used for meta-analyses and $\mathrm{R}$ for additional analyses.22 All meta-analyses were based on the random effects model with the DerSimonian-Laird estimate for variance between studies. For continuous outcomes, we used mean difference if outcomes were measured on the same scale. Otherwise, we calculated Hedges' $g$ as standardised mean difference (SMD: 0.2$<0.5=$ small, $0.5-<0.8=$ moderate, $\geq 0.8=$ large effect) ${ }^{23}$ The pooled SMDs of the quality of life analyses were re-expressed on the global health/QoL scale (item 29 and 30) of the European Organization for Research and Treatment of Cancer (EORTC) QLQ-C30 (0-100, high values=good quality of life). We chose 25.6 as the reference $\mathrm{SD}$, which is the reference value for patients with recurrent/metastatic cancer of different origins. ${ }^{24}$ The procedure of re-expressing SMDs with familiar instruments is described in the Cochrane Handbook (SMD $\times$ standard deviation $_{\text {reference }}=$ value on original scale). ${ }^{19}$ We chose a minimal clinically important difference of 8.1 for the global health/QoL scale based on the regression analyses performed by Osoba and colleagues, which indicated a change of 6.9 (breast cancer, $n=246$ ) and 10.7 (small cell lung cancer, $\mathrm{n}=111$ ) between each category, including the typical category of minimal clinically important difference "a little better." 25 The weighed mean of both slopes results in 8.1, which can be used as minimal clinically important difference.

Risk ratios were calculated for dichotomous outcomes with the Mantel-Haenszel estimate to calculate the variance between studies. Dichotomous data were converted to Hedges' $\mathrm{g}$ (for the depression comparison). ${ }^{23}$ For survival outcomes, we calculated the 
logarithm of hazard ratios and corresponding standard errors using methods described in Parmar and colleagues ${ }^{26}$ when they were not reported. All estimates are presented with $95 \%$ confidence intervals. Significance was set at $\mathrm{P} \leq 0.05$.

We accepted results of cluster randomised controlled trials without additional adjustment if trial authors considered the design properly in their analysis (such as multilevel model). ${ }^{19}$ Otherwise, we took unit of analysis issues into account by adjusting for cluster randomised controlled trials according to the Cochrane Handbook-that is, using intraclass correlation coefficient obtained from authors of primary studies or from literature. ${ }^{19}$ Outcomes were analysed at the point of measurement of the primary outcome as defined in the randomised controlled trials (except for survival and place of death).

We evaluated statistical heterogeneity using $\chi^{2}$ test and $\mathrm{I}^{2}$ statistic $^{23}$ (heterogeneity: 0-40\%=small, $30-60 \%=$ moderate, $50-90 \%=$ substantial, $>75 \%=$ consid erable). ${ }^{20}$ We investigated clinical heterogeneity by prespecified subgroup analyses: cancer versus non-cancer; different non-cancer diseases; early versus not early specialist palliative care (early defined as: Eastern Cooperative Oncology Group 0-2 or Karnofsky index 50-100 or 6-24 months estimated survival or initiation of specialist palliative care within eight weeks after diagnosis of an advanced incurable illness); younger versus elderly patients (age $<60,60-70,>70$ ); and inpatients versus outpatients.

In sensitivity analyses, we used the Paule-Mandel estimate of the variance between studies recently recommended by Veroniki and colleagues. ${ }^{27}$ If studies reported only change in scores or scores after treatment and were included in the same meta-analysis, we evaluated potential differences in a sensitivity analysis. ${ }^{19}$ Studies with extraordinary large effects in either direction were critically checked for flaws and evaluated in sensitivity analyses.

\section{Patient involvement}

We involved no patients in the development of the research question or in the selection of study design and outcome measures. No patients were involved in the conduct of the study. We do not plan to disseminate the results to study participants.

\section{Results}

We identified 3967 records through the database search or other sources (fig 1) and included 10 randomised controlled trials that were published in 12 articles (2454 patients: $1766(72 \%)$ with cancer). The reasons we excluded articles after reading the full text are provided in table B in the appendix.

All trials were performed in hospitals and none in hospices or community settings (table 1 ). The intervention varied across studies. Social workers and chaplains were part of the multiprofessional team in five of the 10 trials (50\%); all 10 studies included a nurse and nine studies (90\%) included a physician. The control intervention was usually described as "standard" or "usual" care. Four studies also provided palliative care in the control arm if this was requested by the patients. One study used a control group with multidisciplinary support ${ }^{28}$ and another provided telephone palliative care. The point in time of measurement of the primary outcome ranged from a few days ${ }^{29-31}$ to six months. ${ }^{32}$ In five studies the primary outcome was assessed at three months (table 1).

All trials provided a "specialist palliative care for all” approach; none initiated integration of specialist palliative care according to the individual needs or symptoms of patients as identified through screening. There were slight sex differences between intervention groups and control groups in the two smallest studies (5/10 $(50 \%)$ v $7 / 10(70 \%)^{29}$ and $37 / 50(74 \%)$ v 21/40 (52\%), ${ }^{32}$ respectively, were women). Across all studies, however, sex was equally distributed (1271/2454 (52\%) were women; table $\mathrm{C}$ in appendix).

Three of the 10 included studies (30\%) were cluster randomised controlled trials. ${ }^{32-35}$ We included two of them in meta-analysis as we considered that the adjustment for clustering was appropriate. ${ }^{33} 34$

The use of the Paule-Mandel estimator did not change any meta-analysis result substantially. We did not identify any systematic differences between change in scores versus scores after treatment if both were combined in a meta-analysis. The only sensitivity analysis presented here evaluates the effect of a study ${ }^{36}$ that had extraordinary small $95 \%$ confidence intervals, resulting in an unusually large SMD.

\section{Risk of bias}

In all studies, the risk of bias was low or unclear for most items (fig 2), but high for a lack of blinding of participants and personnel in nine studies (90\%). Three studies had a high risk for reporting bias because of outcomes that were reported in the protocol but not in the

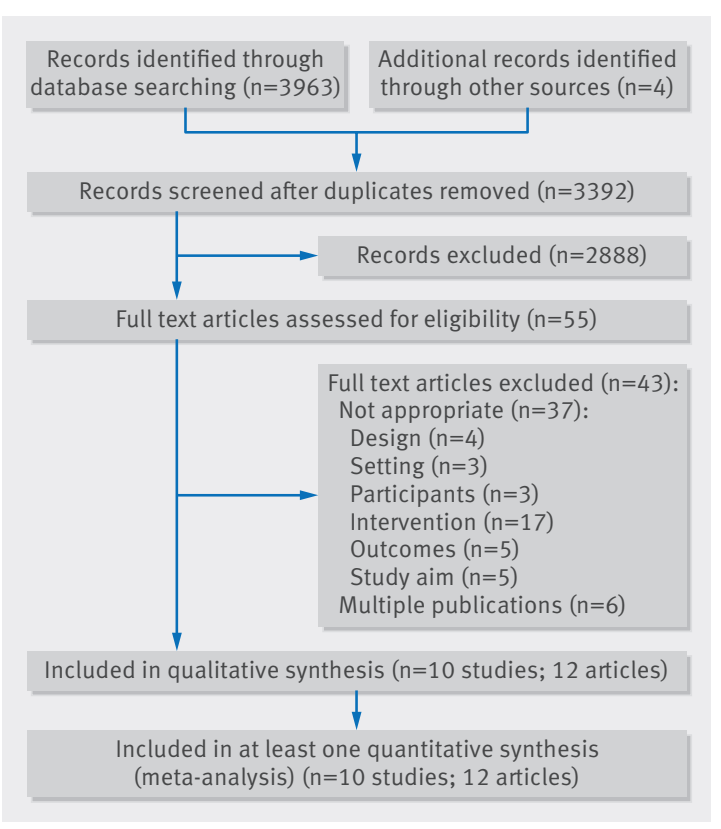

Fig 1 | Flow diagram on inclusion in review of studies on |specialist palliative care 


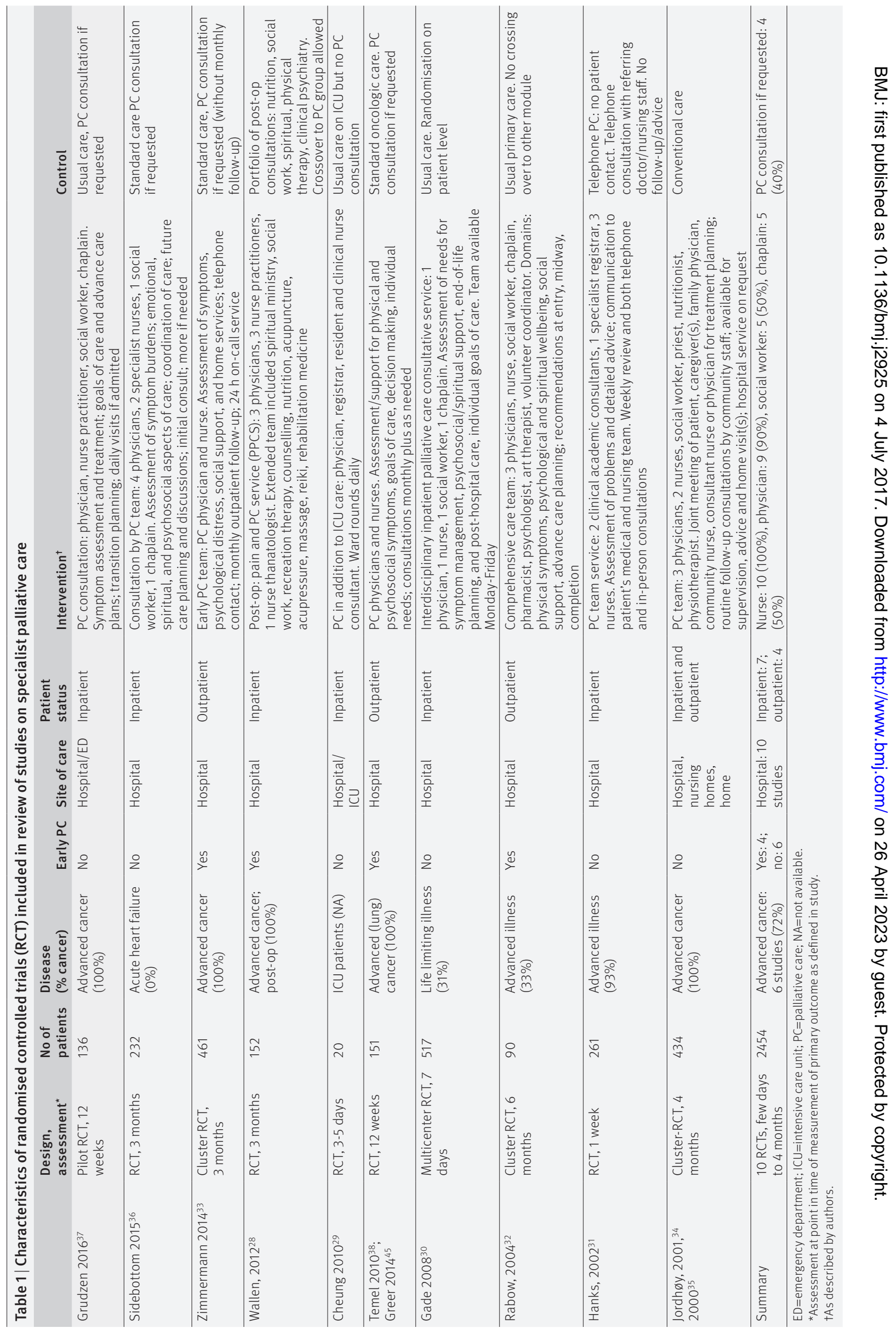


publication ${ }^{3337}$ and because of different information in the protocol and the publication (one instead of three primary outcomes in the protocol). ${ }^{36}$ The risk for attrition (withdrawal or dying) at the point in time of measurement of the primary outcome was balanced (see fig A in appendix). The risk for attrition was slightly lower for standard care in the study by Gade and colleagues. ${ }^{30}$ Rabow and colleagues analysed only patients who competed all surveys. ${ }^{32}$ It is conflicting that the authors also reported deaths (10 in the specialist palliative care group and five in the standard care group) and losses to follow-up (five and four, respectively) during the study in the patient characteristics table. Seven trials (70\%) explicitly mentioned that they had used intention to treat analysis (table $\mathrm{C}$ in appendix). Three explained how missing data were handled. ${ }^{343738}$ Of these three studies, two presented only the available data and not imputed data as the main result. ${ }^{3438}$

\section{Quality of evidence}

We used GRADE to evaluate the quality of evidence of each outcome. This was moderate for quality of life and low for pain. Both outcomes are patient reported and were downgraded because of serious risk of bias from the lack of blinding. In addition, pain was downgraded because of serious imprecision of the $95 \%$ confidence interval (that is, wide range and small effects in both directions, table 2). The quality of evidence for other secondary outcomes is shown in table D in the appendix.

\section{Primary outcome: quality of life}

Eight of the 10 included studies (80\%) measured quality of life. Only two studies used the same questionnaire (EORTC QLQ-C30), while the six other studies used different assessment tools (table 3). The authors used well known and validated questionnaires like EORTC QLQ$\mathrm{C} 30,{ }^{24}$ functional assessment of cancer therapy-general $\left(\right.$ FACT-G) ${ }^{39}$ and lung (FACT-L), ${ }^{40}$ trial outcome index (TOI), ${ }^{40}$ functional assessment of chronic illness therapy-spiritual wellbeing (FACIT-Sp), ${ }^{41}$ Minnesota living with heart failure (MLHF) questionnaire, ${ }^{42}$ and less known validated questionnaires like the modified city of hope patient questionnaire(MCOHPQ) ${ }^{43}$ and the multidimensional quality of life scale (MQOLS) ${ }^{44}$ (table 3).

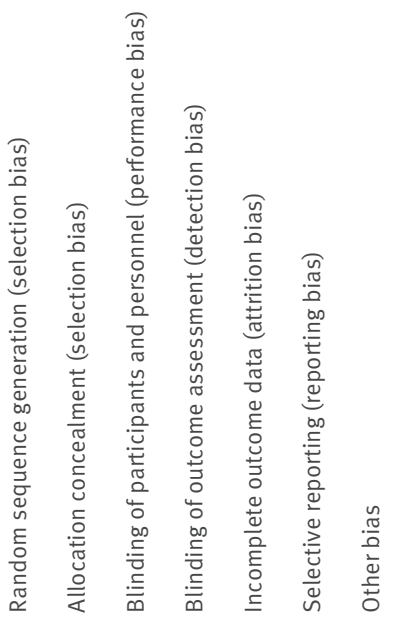

Cheung 2010

Gade 2008

Grudzen 2016

Hanks 2002

Jordhøy 2001, Jordhøy 2000

Rabow 2004

Sidebottom 2015

Temel 2010, Greer 2014

Wallen 2012

Zimmermann 2014

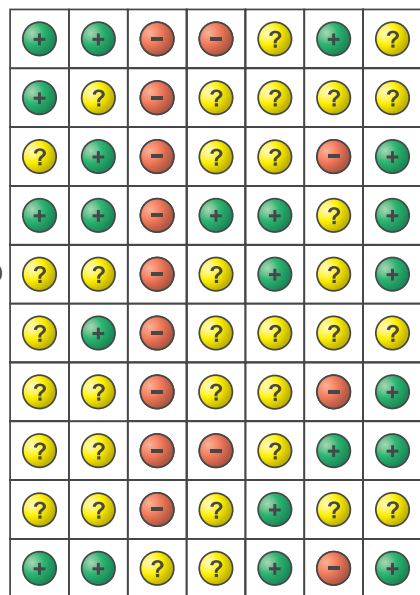

Fig 2 Risk of bias summary in review of studies on |specialist palliative care

Three of the eight studies (38\%) showed a small significant effect ${ }^{36-38}$ and four (50\%) a non-significant effect in favour of specialist palliative care ${ }^{30-33}$ regarding the study specific assessment tools for quality of life (table 3).

We included seven randomised controlled trials in the meta-analysis. Overall, there was a small significant effect in favour of specialist palliative care (SMD 0.16, $95 \%$ confidence interval 0.01 to $0.31 ; n=1218$, six trials, $\mathrm{I}^{2}=38 \%$, moderate quality of evidence; $95 \%$ prediction interval -0.22 to 0.54 ) (fig 3, table 2). The re-expressed

Table 2 | Summary of findings and quality of evidence (GRADE) in review of specialist palliative care (SPC) compared with standard care (StC) for patients with advanced disease

\begin{tabular}{|c|c|c|c|c|c|c|}
\hline \multirow[b]{2}{*}{ Outcomes } & \multicolumn{2}{|c|}{ Anticipated absolute effects ${ }^{\star}(95 \% \mathrm{Cl})$} & \multirow{2}{*}{$\begin{array}{l}\text { Relative } \\
\text { effect } \\
(95 \% \mathrm{Cl})\end{array}$} & \multirow{2}{*}{$\begin{array}{l}\text { No of participants } \\
\text { (studies) }\end{array}$} & \multirow{2}{*}{$\begin{array}{l}\text { Quality of evidence } \\
(\text { GRADE) })^{\dagger}\end{array}$} & \multirow[b]{2}{*}{ Comments } \\
\hline & Risk with StC & Risk with SPC & & & & \\
\hline $\begin{array}{l}\text { Quality of life (follow-up: } \\
\text { median } 3 \text { months) }\end{array}$ & - & $\begin{array}{l}\text { SMD } 0.16 \text { SD higher } \\
(0.01 \text { to } 0.31 \text { higher) } \\
\text { than in StC }\end{array}$ & - & 1218 (6 RCTs) & Moderate ${ }^{\ddagger}$ & $\begin{array}{l}\text { Higher values mean improvement. Effect: } \\
0.2-<0.5=\text { small, } 0.5-<0.8=\text { moderate, } \\
\geq 0.8=\text { large (fig } 3 \text { ) }\end{array}$ \\
\hline $\begin{array}{l}\text { Pain (scale 0-10, } \\
\text { follow-up 3-4 months) }\end{array}$ & $\begin{array}{l}\text { Mean changes } \\
\text { from baseline } \\
-0.49 \text { and } 1.10\end{array}$ & $\begin{array}{l}\text { Mean } 0.38 \text { points lower } \\
(0.82 \text { lower to } 0.06 \text { higher }) \\
\text { than in StC }\end{array}$ & - & $410(3 \mathrm{RCTS})$ & Low $^{\S}$ & $\begin{array}{l}\text { Low values mean improvement. Wallen's } \\
\text { VAS 0-20 divided by } 2 \text { and Jordhøy's VAS } \\
0-100 \text { divided by } 10 \text { for analysis (fig 4) }\end{array}$ \\
\hline
\end{tabular}

$\mathrm{SMD}=$ standardised mean difference; $\mathrm{SD}=$ standard deviation; $\mathrm{RCT}=$ randomised controlled trial; $\mathrm{VAS}=\mathrm{visual}$ analogue scale.

*Risk in SPC (and its 95\% Cl) based on assumed risk in StC and relative effect of intervention (and its 95\% Cl).

TGRADE Working Group grades of evidence. High: very confident that true effect lies close to that of estimate of effect; moderate: moderately confident in effect estimate (true effect is likely to

be close to estimate of effect, but there is possibility that it is substantially different); low: limited confidence in effect estimate (true effect could be substantially different from estimate of

effect); very low: very little confidence in effect estimate (true effect is likely to be substantially different from estimate of effect).

$\neq \mathrm{Q} O \mathrm{E}$ downgraded by one level because of serious risk of bias: blinding of participants and personnel is not possible in SPC studies; assessment of subjective outcome.

$\S Q 0$ E downgraded by one level because of serious imprecision: $95 \% \mathrm{Cl}$ has wide range and includes small effects in both directions. 


\begin{tabular}{|c|c|c|c|c|}
\hline Trial & $\begin{array}{l}\text { Outcome measure* (scale/score } \\
\text { range) }\end{array}$ & $\begin{array}{l}\text { Mean (SD or } 95 \% \mathrm{Cl}) \text { score in } \\
\text { intervention } v \text { control group }\end{array}$ & $\begin{array}{l}\text { Observed } \\
\text { effect } t\end{array}$ & Comments \\
\hline \multirow[t]{2}{*}{ Grudzen $2016^{37}$} & Mean change in FACT G $\neq(0-108)$ & $5.91(16.65) \vee 1.08$ (16.00); $P=0.03$ & + & \multirow{2}{*}{$\begin{array}{l}\text { Results at week 12; QoL at baseline differed } \\
\text { (intervention } 53.56 \text { v control 9.82) }\end{array}$} \\
\hline & Pain not assessed & - & - & \\
\hline \multirow[t]{2}{*}{ Sidebottom $2015^{36}$} & $\begin{array}{l}\text { Mean difference between groups in } \\
\text { MLHF‡ }(0-105)\end{array}$ & $\Delta 3.06$ (2.75 to 3.37); $P<0.001$ & + & \multirow{2}{*}{$\begin{array}{l}\text { Results at month } 3 ; 3 \text { primary outcomes; adjusted } \\
\text { for age, sex, and marital status }\end{array}$} \\
\hline & ESAS $(0-10)$ & $\begin{array}{l}\text { Pain: } \Delta-0.44(-0.13 \text { to }-0.75) \\
P=0.005\end{array}$ & + & \\
\hline \multirow[t]{3}{*}{ Zimmermann $2014^{33}$} & $\begin{array}{l}\text { Change for FACT-spiritual wellbeing } \neq \\
(0-156) \text { : }\end{array}$ & $\begin{array}{l}1.60(14.46) v-2.00(13.56) ; \Delta 3.56 \\
(-0.27 \text { to } 7.40) ; P=0.07 ; d=0.26\end{array}$ & $0 /+$ & \multirow{3}{*}{$\begin{array}{l}\text { Results at month 3; effects at month } 4 \text { greater than } \\
\text { month 3; robust results in sensitivity analyses; } \\
\text { adjusted for cluster and baseline covariates }\end{array}$} \\
\hline & Change for Qual-E (21-105) & $\begin{array}{l}2.33(8.27) \vee 0.06(8.29) ; \Delta 2.25 \\
(0.01 \text { to } 4.49) ; P=0.05 ; d=0.28\end{array}$ & 0 & \\
\hline & Pain not assessed & - & - & \\
\hline \multirow[t]{2}{*}{ Wallen $2012^{28}$} & Quality of life not assessed & - & - & \multirow{2}{*}{$\begin{array}{l}\text { Results at month 3; } 3 \text { primary outcomes but time } \\
\text { of measurement not specified; adjusted for } \\
\text { baseline scores and depression }\end{array}$} \\
\hline & $\begin{array}{l}\text { GPS‡: a) pain intensity }(0-20) \text { b) } \\
\text { pain unpleasantness }(0-20)\end{array}$ & $\begin{array}{l}\Delta: \text { a) }-1.54 ; P=0.14, b)-0.59 \\
P=0.55\end{array}$ & a) $0 /+$, b) $0 /+$ & \\
\hline \multirow[t]{2}{*}{ Cheung $2010^{29}$} & Quality of life not assessed & - & - & \multirow{2}{*}{$\begin{array}{l}\text { Multiple primary outcomes Methodological } \\
\text { limitations }\end{array}$} \\
\hline & Pain not assessed & - & - & \\
\hline \multirow[t]{4}{*}{$\begin{array}{l}\text { Temel } 2010^{38} \text { and Greer } \\
2014^{45}\end{array}$} & TOI $(0-84)$ & $\begin{array}{l}59.0(11.6) \vee 53.0(11.5) ; \Delta 6.0(1.5 \\
\text { to } 10.4) ; P=0.009, d=0.52\end{array}$ & + & \multirow{4}{*}{ - Results at week 12; adjusted for baseline scores } \\
\hline & FACT-lung (0-136) & $\begin{array}{l}98.0(15.1) \vee 91.5(15.8) ; \Delta 6.5(0.5 \\
\text { to } 12.4) ; P=0.03, d=0.42\end{array}$ & + & \\
\hline & FACT-lung subscale (0-28) & $\begin{array}{l}21.0(3.9), 19.3(4.2) ; \Delta 1.7(0.1 \text { to } \\
\text { 3.2); } P=0.04, d=0.41\end{array}$ & $0 /+$ & \\
\hline & Pain not assessed & - & - & \\
\hline \multirow[t]{2}{*}{ Gade $2008^{30}$} & MCOHPQ $\ddagger(0-10)$ & $6.4(2.3) \vee 6.3(2.1) ; P=0.78$ & $0 /+$ & \multirow{2}{*}{$\begin{array}{l}\text { Assessed } 2 \text { weeks after discharge; median days of } \\
\text { stay: } 7 ; 5 \text { primary outcomes; no adjustments }\end{array}$} \\
\hline & Pain not assessed & - & - & \\
\hline \multirow[t]{5}{*}{ Rabow $2004^{32}$} & MQOLS-CA (0-100) & $69.7 \vee 65.4$ & $\mathrm{NA} /+$ & \multirow{5}{*}{$\begin{array}{l}\text { Results at } 6 \text { months; primary outcome and time } \\
\text { not stated; no P values at month 6; no SDs; } \\
\text { adjusted for baseline scores }\end{array}$} \\
\hline & BPI pain intensity $(0-10)$ & & & \\
\hline & Average & $4.8 v 4.9$ & $\mathrm{NA} /+$ & \\
\hline & Worst & $5.9 \vee 5.5$ & $\mathrm{NA} /-$ & \\
\hline & Least & $2.7 \vee 3.9$ & $\mathrm{NA} /+$ & \\
\hline \multirow[t]{2}{*}{ Hanks $2002^{31}$} & EORTC QLQ-C30 $(0-100)$ & $\begin{array}{l}37.1->47.3(P<0.001) \vee 39.3->45.5 \\
(P<0.044) ; \Delta 2.35(-3.7 \text { to } 8.4) \\
P=0.45\end{array}$ & $0 /+$ & \multirow{2}{*}{$\begin{array}{l}\text { Results at week } 1 ; 4 \text { primary outcomes; } 19 / 86 \\
\text { ( } 22 \%) \text { switched to intervention, } 10 \text { in week } 1 \text {; } \\
\text { - adjusted for baseline scores }\end{array}$} \\
\hline & Pain not assessed & - & - & \\
\hline \multirow[t]{2}{*}{ Jordhøy $2001,342000^{35}$} & $\begin{array}{l}\text { EORTC QLQ-C30-global health } \neq \\
(0-100) \text { : }\end{array}$ & $50(25.61) \vee 53(21.95)$ & $\mathrm{NA} /-$ & \multirow{2}{*}{$\begin{array}{l}\text { Results after } 4 \text { months; } 4 \text { primary outcomes; no } \\
\text { adjustment; authors contacted for SD values }\end{array}$} \\
\hline & $\begin{array}{l}\text { EORTC QLQ-C30-symptom scaleł } \\
(0-100)\end{array}$ & Pain: 41 (33.90) v 37 (31.49) & $0 /-$ & \\
\hline \multicolumn{5}{|c|}{ 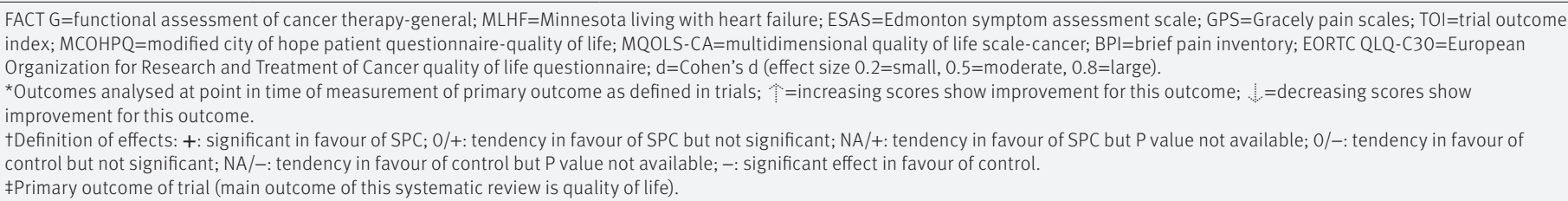 } \\
\hline
\end{tabular}

$\ddagger$ Primary outcome of trial (main outcome of this systematic review is quality of life).

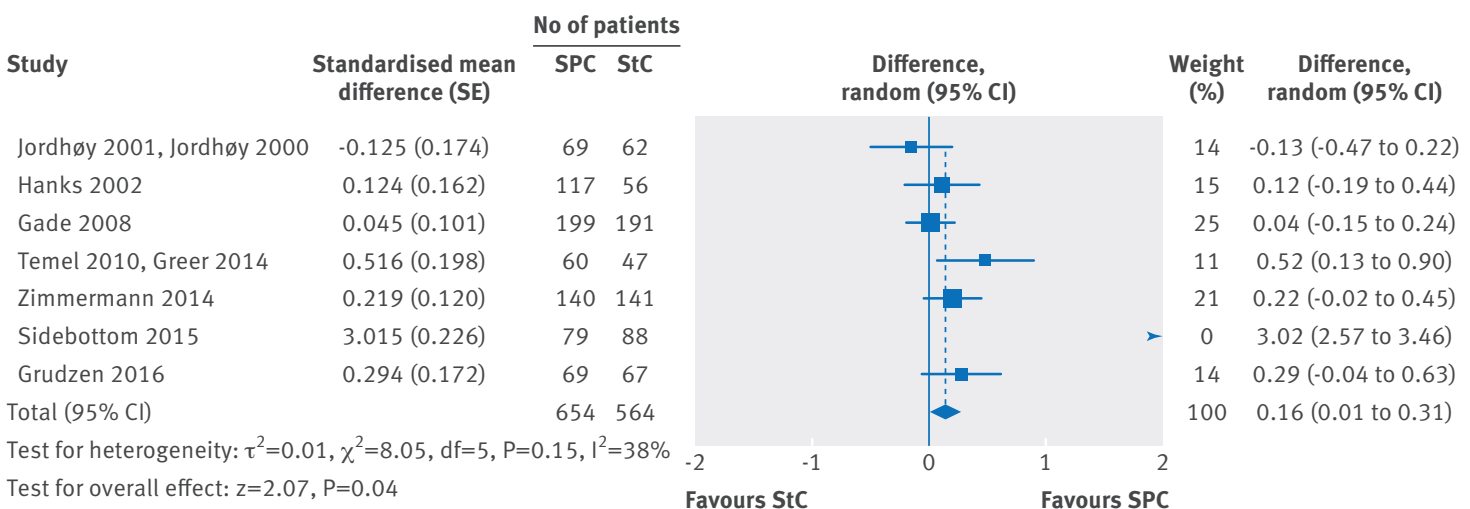

Fig 3 | Effect on total quality of life (primary outcome) in review of studies on |specialist palliative care (SPC) versus standard care (StC) (study by Sidebottom et al ${ }^{36}$ was not included in meta-analysis) 


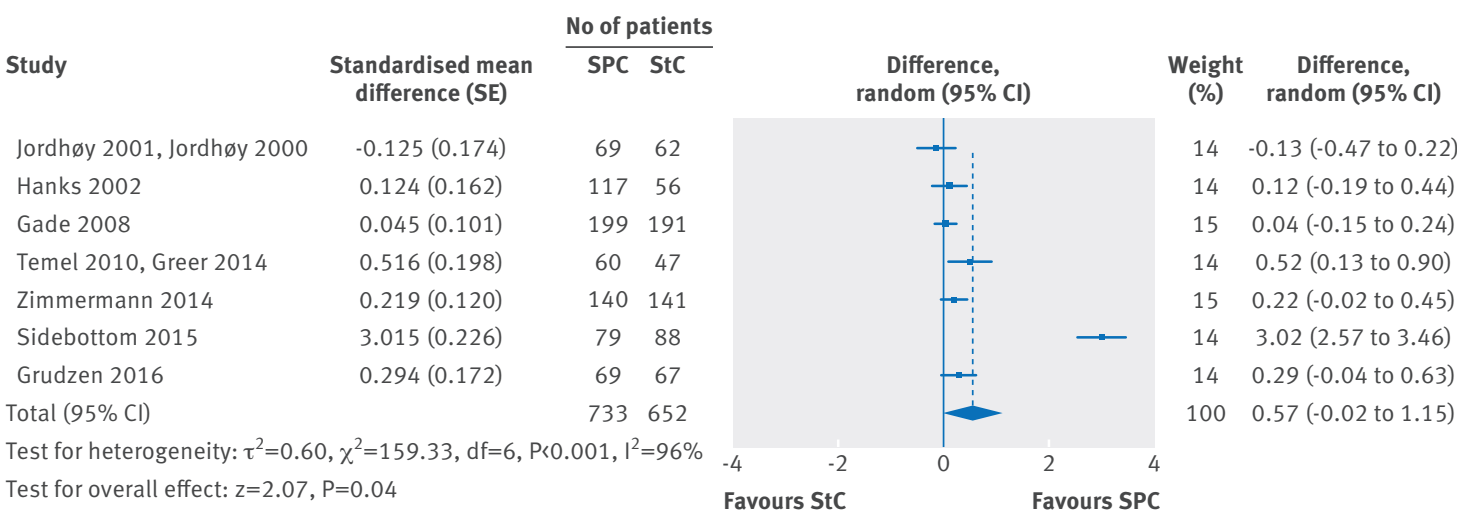

Fig 4 | Effect on total quality of life (primary outcome) in review of studies on |specialist palliative care (SPC) versus standard care (StC) (including Sidebottom et al ${ }^{36}$ )

effect on the EORTC QLQ-C30 global health/QoL scale was 4.1 ( 0.3 to 8.2$)$.

In the sensitivity analysis (in which we included a study ${ }^{36}$ with a critically large effect), the effect estimate was much larger, though the $95 \%$ confidence interval also increased substantially because of excessive heterogeneity between studies (SMD: 0.57, 95\% confidence interval -0.02 to $1.15 ; n=1385$, seven trials, $I^{2}=96 \%$; fig 4). An effect of 14.6 ( -0.5 to 29.4 ) was observed when the SMD was re-expressed on the EORTC QLQ-C30 global health/QoL scale.

The effect in favour of specialist palliative care was marginally higher for patients with cancer (SMD: 0.20, $95 \%$ confidence interval 0.01 to 0.38 ; $n=828$, five trials; fig 5 ) and highest for early care (0.33, 0.05 to 0.61 ; $\mathrm{n}=388$, two trials; fig 6). The re-expressed effects for the latter on the EORTC QLQ-C30 global health/QoL scale were 5.1 (0.3 to 9.7) and 8.5 (1.3 to 15.6), respectively.
Results of a sensitivity analysis of early versus not early specialist palliative care (including the Sidebottom study ${ }^{36}$ ) and a subgroup analysis by age are provided in the appendix (figs $B$ and $C$ ).

\section{Pain}

Four studies evaluated pain as outcome. Two numerical rating scales, ${ }^{3236}$ a visual analogue scale, ${ }^{28}$ and the combination of two transformed verbal rating scales (range 0-100) ${ }^{34}$ were used to assess pain (table 3). One study showed contradictory results and could not be included in the meta-analysis because it did not provide standard deviations. ${ }^{32}$ We included three studies 283436 in the meta-analysis after we linearly transformed the values of two of them ${ }^{2834}$ to a scale ranging from 0 to 10 (higher values=more pain) (fig 7). Compared with standard care alone, the pooled effect for specialist palliative care showed a small but non-significant effect

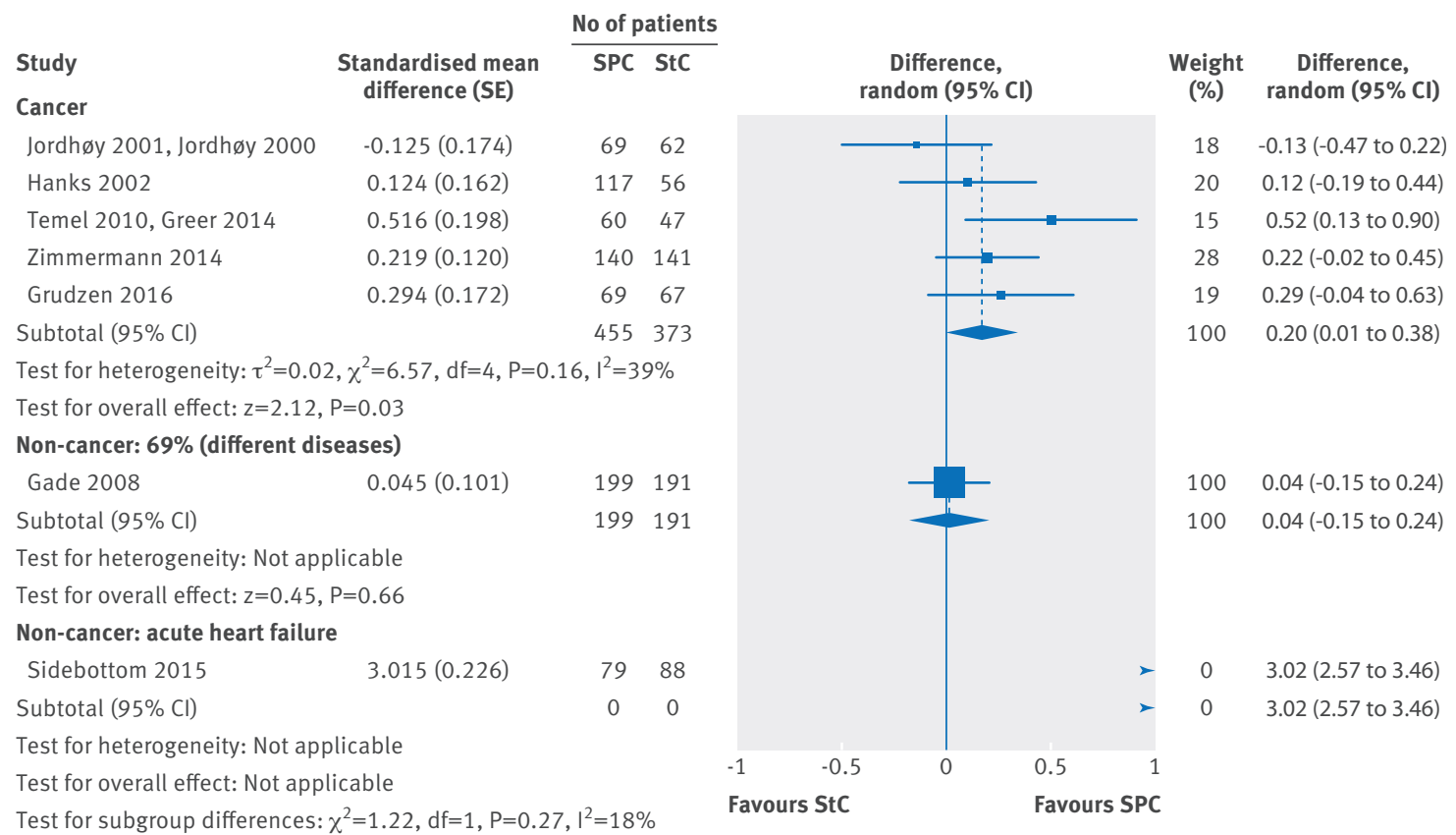

Fig 5 Effect on quality of life (primary outcome) in review of studies on |specialist palliative care (SPC) versus standard care (StC) (study by Sidebottom et $\mathrm{al}^{36}$ was not included in meta-analysis). Subgroup analysis in patients with and without cancer 


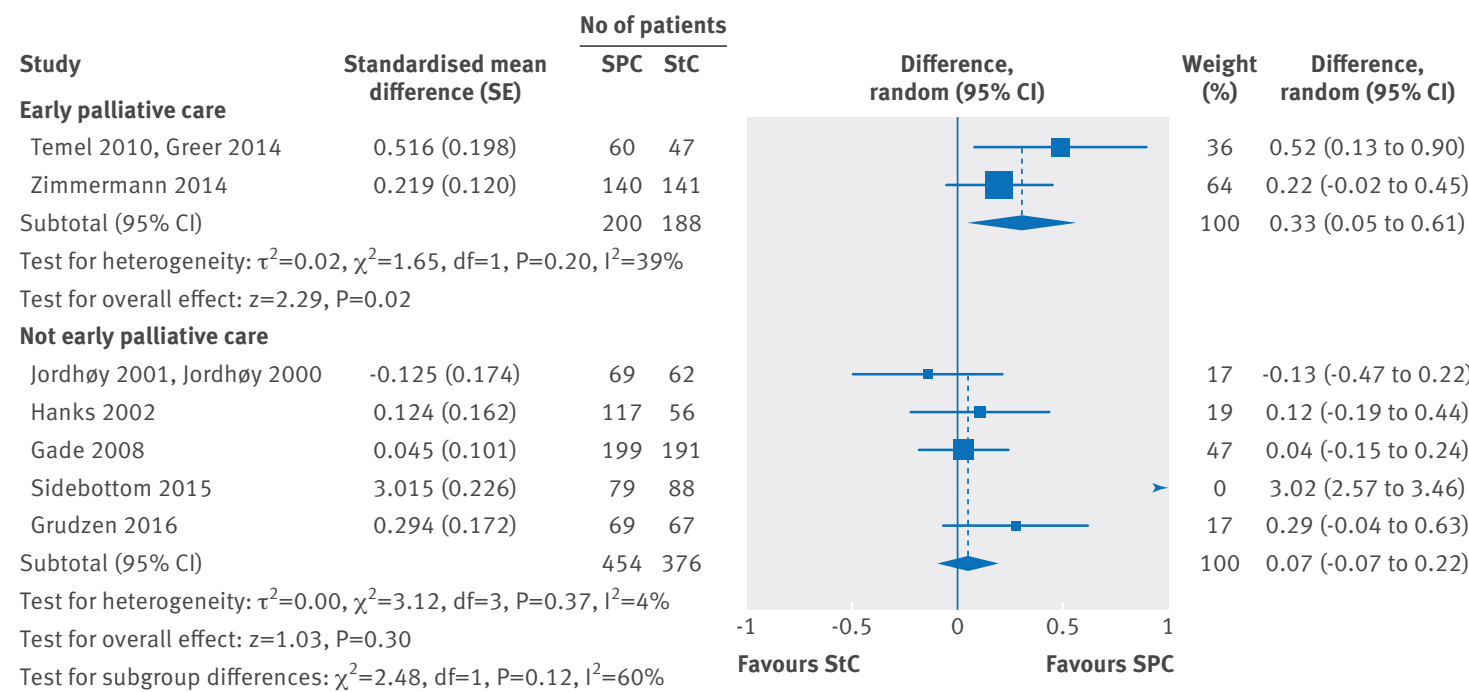

Fig 6 | Effect on quality of life (primary outcome) in review of studies on |specialist palliative care (SPC) versus standard care $(\mathrm{StC})$ (excluding Sidebottom et $\mathrm{al}^{36}$ ). Subgroup analysis in patients who received SPC early $v$ not early

$(-0.38,95 \%$ confidence interval -0.82 to $0.06 ; n=410$, three studies, $\left.\mathrm{I}^{2}=23 \%\right)$. The quality of evidence was low and downgraded because of lack of blinding and wide 95\% confidence intervals with effects in both directions.

Other outcomes, subgroup analyses, and additional information

The results for other secondary outcomes were inconclusive. Secondary outcomes and subgroup analyses are shown in the appendix (text, tables D-F, and figs D-J). Ongoing studies are reported in table $\mathrm{G}$ in the appendix and differences between protocol and publication are shown in table $\mathrm{H}$.

\section{Discussion}

Summary of the findings

Specialist palliative care and its early integration might have a small effect on the quality of life of patients with cancer and without cancer, based on moderate quality of evidence. The effects on quality of life were most pronounced for patients with cancer and early integration of specialist palliative care. Notably, the results for pain and other secondary outcomes were inconclusive. Because of the obvious equivocal nature of the studies included in our review, special attention must be paid to the meticulous discussion of these findings. For this, we have provided detailed descriptions of the studies' characteristics (strengths and weaknesses) along with specific considerations concerning methodological aspects of the meta-analysis.

\section{What the review adds}

This systematic review differs from previous publications $^{5-7}$ in several aspects. These include the clear definition of inclusion criteria, clarity and extent of the provided results, a priori specified subgroup analyses (such as cancer, early specialist palliative care), and interpretability.

We performed meta-analyses and sensitivity analyses for key outcomes. In contrast with previous works, ${ }^{5-7}$

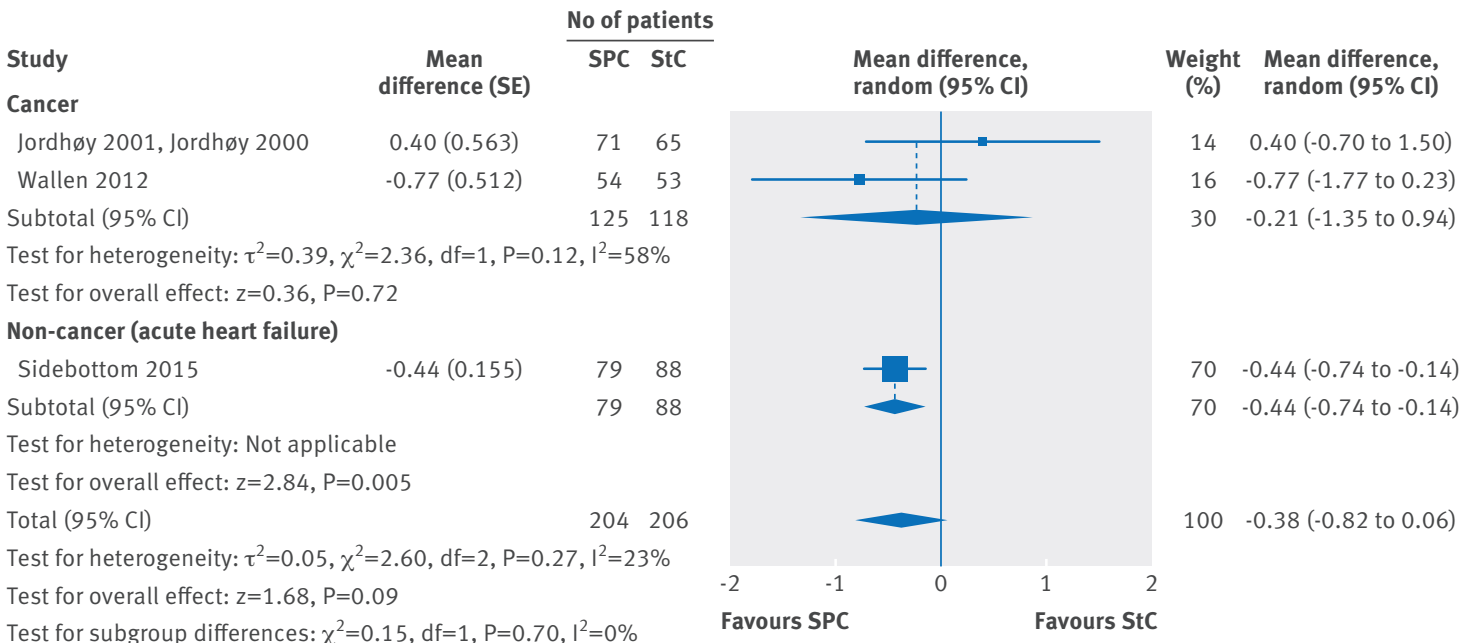

Fig 7 | Effect on pain (secondary outcome; range $0-10$ ) in review of studies on specialist palliative care (SPC) versus standard care (StC) 
we provide detailed information about effect size, confidence intervals, significance, and a prediction interval for the main result. The SMD had to be calculated for the quality of life meta-analysis because different tools were used by the authors. We provided results on the original scales (table 3) and as SMD in the meta-analysis. Because understanding of the effect as SMD might be not intuitive, we re-expressed the SMD on the EORTC QLQ-C30 global health/QoL scale. The confidence intervals of the latter overlapped the minimal clinically important difference of 8.1 for all comparisons, indicating that specialist palliative care might have a clinically meaningful effect. Also, of utmost importance is a precise definition of inclusion and exclusion criteria for specialist palliative care interventions, and the discussion of the methodological quality of the trials (see below). This could have important implications for the conclusions drawn for practice and policy. ${ }^{12}$

\section{Strengths, weaknesses, and characteristics of the included studies}

As is the case in most research settings of palliative care, the included trials differed largely in several aspects, such as the population studied, the outcomes chosen, the clinical setting, and the duration of the study.

The oldest trial, published by Jordhøy and colleagues in 2000-01, included only patients with advanced cancer. $^{3435}$ This cluster randomised controlled trial is the only trial that provided specialist palliative care for inpatients and outpatients, and the care team relied on a comparably large and multiprofessional workforce including three physicians, two nurses, social worker, priest, nutritionist, and physiotherapist. The team scheduled routine follow-up visits with the community staff and was available for home visits, visits in nursing homes, and visits in the hospital. The primary outcomes (Jordhøy and colleagues defined multiple primary outcomes) included quality of life measured on a cancer specific questionnaire (EORTC QLQ-C30) and were assessed after four months, and patients were even followed for up to six months. Though it is one of the three largest trials (434 patients randomised), only around one of three patients completed the trial, mainly because of the high mortality in this population with advanced cancer (table F in the appendix), which might impede intention to treat analysis.

The second oldest trial we included was that of Hanks and colleagues ${ }^{31}$ in 2002. The so called imPaCT study was not restricted to patients with cancer, but in 243 of the 261 randomised inpatients (93\%) cancer was the leading disease. The specialist palliative care team consisted of nurses and physicians. Interestingly, in contrast with all other studies, all patients in the control group also received palliative care, but this was limited to telephone advice. Even though, this could have led to an underestimation of the true effect of the active intervention. As results were obtained after the first week, it did not allow for capturing long term effects of specialist palliative care. By week one, data were already unavailable for more than one in four patients who was initially randomised (10 of these had switched from the control to the intervention group). Patients were reported as missing because they were too ill, tired, or just not available. Though attrition is always an issue in clinical research in patients with advanced progressive disease, such a high dropout is surprising after just one week. We do not know whether this could have led to underestimation or overestimation of the true effect of the intervention.

In 2004 Rabow and colleagues published results of a relatively small cluster randomised controlled trial $(n=90)$ studying the effects of specialist palliative care on patients with $(n=30,33 \%)$ and without cancer who were cared for in hospital outpatient clinics. ${ }^{32}$ The results were obtained after six months, so this study reported long term effects. The care team consisted of many different professions (physicians, nurse, social worker, chaplain, pharmacist, psychologist, art therapist, volunteer coordinator) and provided recommendations on study entry in the middle and the end of the trial. Problematically, results were reported with the number of randomised patients (50 in the intervention group and 40 in the control group), even though considerable dropout was reported after 12 months (table F in the appendix). Also problematic, no P values or standard deviations were reported at six months and are unavailable because of death of the author and loss of the original data. We could not therefore include this trial in the meta-analysis for the primary outcome (quality of life).

In 2008, Gade and colleagues published results of the biggest trial included in our review. ${ }^{30}$ As in the study of Rabow and colleagues ${ }^{32}$ this multicentre randomised controlled trial included patients with (159/512, 31\%) and without cancer, but in contrast, included only inpatients. Here, the specialist palliative care team was also able to provide care from four professions (physician, nurse, social worker, chaplain). Key outcomes were obtained two weeks after discharge from the hospital. At this time, dropout was 29\% (81/280) in the intervention group and 19\% (46/237) in the control group, mainly because of a larger number of deaths in the intervention group. This might impede intention to treat analysis but was mainly due to premature death of the patients (median survival between 30 and 36 days in the two groups). Overall survival (that is, during admission to hospital and discharge) did not differ significantly.

Temel and colleagues carried out a randomised controlled trial with 151 outpatients with lung cancer who received early palliative care provided by a specialist team (only two professions: physician and nurse) on a regular basis (intervention) or at the request of the oncology team (control). ${ }^{3845}$ They also reported significant dropout at 12 weeks (17/77 (22\%) in the intervention group and $27 / 74$ (36\%) in the control group), when the primary endpoint was assessed. The main analysis of the study was based on complete cases. The authors stated that "last observation carried forward" was used to deal with missing data in a sensitivity analysis and Temel and colleagues assume that this may lead to 
underestimation of the intervention's true effect. Though this method is widely criticised, ${ }^{46}$ it could still provide a conservative approach in a deteriorating population. ${ }^{47}$

The 2010 study by Cheung and colleagues ${ }^{29}$ differed considerably from other included studies. Some of the study characteristics not only make it difficult to compare the findings to other studies but are also problematic from the methodological view. In this randomised controlled trial, patients in the intensive care unit were included if the duty intensivist deemed it appropriate that a "do not resuscitate" order be written for the patient. In the intervention group, patients were seen by a specialist palliative care team (nurse, physician, registrar). Only 20 patients were randomised, and most (16) died in hospital. It is unclear how many of them had cancer. Quality of life measures were not obtained (we did not include this study in meta-analysis of our primary outcome), but other outcomes were recorded on death or discharge. Despite the small study sample and the short duration of the trial (at least two days), data were unavailable for 11 of the 20 patients. Still, the authors attempted intention to treat analysis without providing information about how they dealt with missing data.

In the randomised controlled trial by Wallen and colleagues, ${ }^{28}$ patients with advanced cancer who were admitted for surgery were randomised postoperatively to be followed by a pain and specialist palliative care team (nurse and physician, but other closely associated team members on demand including social work, chaplaincy, reiki, nutrition, etc). The team held a 24 hour on-call service and provided monthly follow-up. The authors failed to obtain quality of life data, and the definition of the primary outcome was not clear. The authors obtained data at three, six, and 12 months, but it is unclear which time they consider most relevant. Dropout was around 30\% after three months (24/76 (32\%) in intervention group, 23/76 (30\%) in control group). The author did not mention an imputation method for dealing with missing data.

More recently, Zimmermann and colleagues performed an elaborate cluster randomised controlled trial, the second largest trial included in this review $(\mathrm{n}=461) .{ }^{33}$ Patients with cancer received either standard care with specialist palliative care consultations as requested by the oncology team (control) or regular visits (at least monthly plus on demand) and a 24 hour on call service of a professional specialist palliative care team (physician and nurse). Patients were followed closely; most patients had four or more visits with the team. In addition, the team was available for inpatient visits if the patients were admitted to hospital. Though the dropout rate for completing the FACIT-Sp was high (180/461 (39\%) after three months), it similar in both groups (see also table $\mathrm{F}$ in the appendix). Approaches for dealing with missing data (last observation carried forward, complete case evaluation, and multiple imputations) were described in detail, and results were robust.

Sidebottom and colleagues ${ }^{36}$ published results of a recent randomised controlled trial that is quite different from the others included in the review. The authors provided palliative care for patients with heart failure, which is a rather neglected issue. ${ }^{48}$ The study was adequately powered and included 232 inpatients who received specialist palliative care if requested by the cardiology team (control) or a mandatory initial visit with the well equipped team (four professions). If necessary, further appointments were scheduled. Interestingly, overall survival of patients in the intervention group was shorter, though this was not significant. Dropout was 32\% (79/116) in the intervention group and $24 \%(28 / 116)$ in the control group after three months, with 14 and five deaths, respectively. The authors did not give information on how they dealt with missing data. More bothersome are issues around the assessment of quality of life, which was performed with the Minnesota living with heart failure questionnaire (MLHF). Sensitivity analysis for this trial showed that though the treatment effect was modest (mean difference of 3.06 on MLHF, range-105) the quality of life estimate was extremely large (SMD 3.02) because of small variation of values (authors were contacted for verification). This extreme treatment effect would have severely affected the findings of the meta-analysis and resulted in extreme heterogeneity (that is, $I^{2}=96 \%$, fig 4 ). We therefore excluded the study from our primary quality of life meta-analysis.

Grudzen et $\mathrm{al}^{37}$ published the most current randomised controlled trial that we included. In this elaborate adequately powered trial $(n=136)$, the authors included patients with cancer but initiated specialist palliative care consultation (intervention group) only for those patients who were referred to the emergency department (control was consultation on request of the emergency physicians). After 12 weeks, quality of life of patients receiving specialist palliative care was significantly and clinically better. Missing data, however, were dealt with by carrying forward baseline measures to perform intention to treat analysis.

\section{Potential reasons for overestimation and underestimation of effects}

Lack of blinding in specialist palliative care interventions might have accounted for overestimation of effects because of performance and detection bias. In addition, we could not include two studies with inconclusive or even negative results for specialist palliative care ${ }^{30} 32$ in the survival analysis and other meta-analyses because of inadequate reporting of data (authors were contacted).

Temel and colleagues reported a large number of missing values because of early deaths of patients. ${ }^{38}$ They observed similar results, however, in a complete case analysis and with the "last observation carried forward" approach. They point out that this method might lead to underestimation of the true effect, which in this study would therefore also apply for the complete case analysis.

Additionally, the implementation of a randomised controlled trial by itself leads to increased recognition of the specialist palliative care team and palliative care issues in 
general. ${ }^{7}$ This increases referral to specialist palliative care and recognition of palliative care needs in the control group, though the number of contacts by the palliative care team in the control group was usually not reported in the included studies. Moreover, some of the included studies even provide enhanced palliative care support in addition to standard care in the control arm. For example, Hanks and colleagues provided palliative care telephone consultations for the standard care group. ${ }^{31}$

\section{Implications for practice and policy}

"Specialist palliative care for all" versus "as needed" Our findings might be surprising and even disappointing for many advocates of palliative care because the reported effect sizes are smaller than many have expected. Yet, we strongly believe that the most important reason for the small effect size on quality of life and the inconclusive findings for the secondary outcomes could be the care approach used in the trials. All trials provided a "specialist palliative care for all" approach and neglected the potential role of general palliative care. As a result, all patients at certain stage of a certain disease were referred to specialist palliative care. For example, in the study of Temel and colleagues all patients with non-small cell lung cancer stage IIIb and IV were referred to the intervention, even if they did not have major symptoms (such as pain, anxiety, dyspnoea, etc) or other distressing conditions such as spiritual or social problems. ${ }^{38}$ Meanwhile, many from the specialty of palliative care question the feasibility, practicability, and efficiency of this approach. ${ }^{1}$ Quill and Abernethy reported that it is not feasible to refer all patients at the palliative stage of their disease to specialist teams. ${ }^{1}$ Such teams cannot be made available for so many patients because of resource allocation issues. ${ }^{149}$ The authors also point to the obligation of every physician to hold general skills in palliative care. Physicians must be capable and willing to deal with basic needs for palliative care but also refer those patients with complex needs to specialist palliative care.

We strongly support such an approach ("general palliative care for all plus specialist palliative care as needed"). This model recognises the importance of general palliative care (and interventions that will strengthen palliative care) as well as the necessity to provide specialist care to patients for whom general palliative care is not enough. Yet, we must emphasise a missing link. In our view, this link is routine structured screening for needs of all patients for palliative care as reported by the patients themselves. Such routine screening (for example, for symptom burden) is known to increase quality of life in these patients and reduce emergency admissions ${ }^{50}$ and could be a practical trigger to identify those who need specialist palliative care. ${ }^{51}$ It is beyond the scope of this review to recommend a certain tool, but it is obvious that such a questionnaire (paper and pencil or electronic) must at the minimum contain the main symptoms (such as pain, anxiety, dyspnoea) reported by patients themselves.

Given the hypothesis, that "specialist palliative care as needed" is more effective, this would mean that the effects of specialist palliative care reported by the studies included in our review might have been underestimated because patients without such needs were included in the intervention group (potential non-responders) and, as reported by Zimmermann and colleagues, ${ }^{7}$ the implementation of trials leads to an increased recognition of palliative care issues and an enhanced use of specialist palliative care in the standard care group.

\section{Patients with and without cancer}

The American Society of Clinical Oncology (ASCO) recommends the integration of (specialist) palliative care early in the course of the disease. ${ }^{6}$ Our findings support this recommendation (figs 5 and 6). The meta-analyses for patients with heart failure indicate a large effect on quality of life (fig 4). Conclusions should be drawn carefully, however, because of the methodological aspects of the heart failure study ${ }^{36}$ that were discussed above. Future studies are needed to reproduce these quality of life findings.

\section{Multiprofessional team}

Our definition for specialist palliative care was strict. For example, we chose the multiprofessional team approach as major prerequisite based on discussions with another working group in the specialty. ${ }^{712}$ This could be problematic because it excludes excellent randomised controlled trials, such as the trial by Maltoni and colleagues ${ }^{52}$ (increased quality of life) or Bakitas and colleagues ${ }^{53}$ (increased survival). In these and other similar trials (table B in the appendix), the palliative care intervention comprised mainly one profession. Interestingly, in the physician led palliative care intervention of Maltoni and colleagues, 52 the reported effects on quality of life were restricted to physical domains. In preparation of the protocol, we had numerous discussions within the working group and also with other working groups ${ }^{12}$ concerning this question. Yet, we decided to stick to the definition of Zimmerman and colleagues ${ }^{7}$ because this had so far been the only systematic review on specialist palliative care. More important, most members of our working groups believed that according to the WHO definition and the general understanding of palliative care, a multiprofessional team should be mandatory, particularly in specialised palliative care.

\section{Limitations and implications for future research} Several limitations of this review must also be noted. Some might be avoidable in the future if researchers in the specialty would agree on basic recommendations.

\section{Endpoints, statistics, risk of bias}

- Quality of life is the main goal of palliative care ${ }^{4}$ and therefore the primary outcome of this review. Surprisingly, not all trials assessed it. We suggest that researchers should implement at least one validated quality of life measure in future randomised controlled trials (table $\mathrm{G}$ in the appendix) 
- Because of the different measures of quality of life, we had to use the SMD for the meta-analysis of this primary outcome. The tools were variable, including general tools (EORTC QLQ-C30, FACT-G) and disease specific (TOI, MLHF) and domain specific (FACIT-Sp) tools. As a pragmatic approach, we re-expressed the pooled SMDs for the EORTC global health/QoL scale and provided a minimal clinically important difference for the clinical interpretation. Ideally, future studies should use a consensus based measure of quality of life that assesses as many domains as possible (physical, psychosocial, spiritual), is validated (in many languages), and is change sensitive. Though the commonly used FACT-G questionnaire 333738 and the EORTC QLQ-C30 questionnaire have been used successfully in many trials, they are restricted to patients with cancer. The ideal tool would be disease independent. It is beyond the scope of this manuscript to recommend a specific tool

- Risk of bias was high in most studies, relating to moderate quality of the assessable evidence of quality of life. The main problem of trials in specialised palliative care that contributes to this is blinding of personnel and participants. This problem is so far unsolved and will probably remain a main challenge in assessing complex palliative care and specialised palliative care interventions

- Some minor deviations from the registered review protocol are reported in table $\mathrm{H}$ in the appendix

- Typically, many patients in specialised palliative care studies will die before the time point of the primary quality of life analysis. As recommended by Shih (2002), ${ }^{54}$ patients dying before the final analysis should be included in the analysis by imputing the worst value. For patients lost to follow-up for other reasons, more advanced methods to impute missing values, such as multiple imputation, should be used instead of the inferior method of last observation carried forward.

\section{Fundamental challenges for conducting trials in palliative care}

The scarcity of the evidence we found for our review might be surprising, but several fundamental issues impede the conduct of interventional trials in the specialty of palliative care. For example, from the public and ethical perspective it might be questionable to provide a palliative care intervention (here specialised palliative care) only for those patients who are in the intervention group, even though palliative care should be available for all patients. This refers to the ethical principle of equipoise-that is, an intervention that is thought to be beneficial should not be withheld from patients in the control arm of a trial. ${ }^{55}$ In the palliative care setting, this is a dilemma. One does not want to restrict ideal palliative care to those patients who are in the intervention group of a clinical trial. Yet, in practice, such trials are the only means to provide routine palliative care for patients in many centres because palliative care services are not sufficiently used. One of the main reasons is that non-palliative care physicians often hesitate to refer to palliative care services. Therefore, trials are needed to provide the necessary evidence to overcome these barriers to referrals.

In addition, specialised palliative care is a complex intervention that involves teamwork of different professions. To develop feasible and effective interventions in different settings, a structured approach is required. The MRC Framework ${ }^{56}$ provides concise guidance for such a project. Such a complex and time consuming process requires resources that many research groups around the world cannot rely on. It can be assumed that many of them fail somewhere along the way between identifying the research question, writing a meaningful protocol, and developing a meaningful intervention.

Concerning the scarcity of the available evidence, we are optimistic for the future. Currently, many protocols for randomised controlled trials assessing the effect of specialised palliative care have been registered (table $G$ in the appendix). We believe that updates to our review will be able to include a larger number of randomised controlled trials in a future meta-analysis.

\section{Conclusion}

The integration of specialised palliative care was associated with a small effect on quality of life, whereas the results for pain and other secondary outcomes were inconclusive. The effect on quality of life might be more pronounced for patients with cancer and for those who received specialised palliative care early. This effect was observed even though all trials also provided specialised palliative care to patients who did not have symptoms nor had any other needs for palliative care. Instead, it was initiated according to diagnosis and stage of disease. Moreover, the true effects of specialised palliative care might have been underestimated because of various methodological issues. We hypothesise that specialised palliative care could be most effective if it is provided early and if it identifies patients with unmet needs through screening ("care as needed"). We hope that the discussion of the importance of general palliative care and the detailed description of shortcomings of the included studies will increase the quality of further clinical research in the specialty of palliative care.

We thank Cinzia Brunelli (statistician, palliative care, pain therapy and rehabilitation unit, Fondazione IRCCS Istituto Nazionale Tumori) for providing statistical data of the study from Jordhøy and colleagues ${ }^{3435}$; Abbey Sidebottom (division of applied research, Allina Health,

Minneapolis, Minnesota) for re-running the change score regression models and confirming their results; and Mayang Mayang, graduate assistant at the department of palliative care, University Medical Center Freiburg, for her help with data extraction and proofreading. Contributors: JG and WS contributed equally. JG had the idea for the work, wrote the protocol, extracted data, evaluated quality of evidence (GRADE), wrote the abstract, introduction, discussion, and conclusion section of the manuscript, and critically revised the entire manuscript. WS extracted data, evaluated quality of evidence (GRADE) and economic analyses, conducted meta-analyses, wrote methods and results; and critically revised and finalised the manuscript. JJM and GA wrote and critically revised the protocol, supervised application of Cochrane standards and GRADE, and critically revised the manuscript. CX critically revised the protocol and manuscript. SS wrote the protocol and supervised the economic analysis. GS wrote the protocol,

supervised meta-analysis and all other statistical analyses, and did a detailed revision of methods and results section of the manuscript. GB critically revised the protocol and manuscript. JG is guarantor. 
Funding: This review was funded by the German Ministry for Education and Research (BMBF), Germany, Grant No. 01 KG1408. The funder of the study had no role in study design, data collection, data analysis, data interpretation, or writing of the report. The corresponding author had full access to all the data in the study and had final responsibility for the decision to submit for publication.

Competing interests: All authors have completed the ICMJE uniform disclosure form at www.icmje.org/coi_disclosure.pdf and declare: no support from any organisation for the submitted work; no financial relationships with any organisations that might have an interest in the submitted work in the previous three years; no other relationships or activities that could appear to have influenced the submitted work.

Ethical approval: Not required.

Data sharing: No additional data available

Transparency: The lead authors (JG, WS) affirm that the manuscript is an honest, accurate, and transparent account of the study being reported; that no important aspects of the study have been omitted; and that any discrepancies from the study as planned have been explained.

This is an Open Access article distributed in accordance with the Creative Commons Attribution Non Commercial (CC BY-NC 4.0) license, which permits others to distribute, remix, adapt, build upon this work non-commercially, and license their derivative works on different terms, provided the original work is properly cited and the use is non-commercial. See: http://creativecommons.org/licenses/ by-nc/4.0/.

1 Quill TE, Abernethy AP. Generalist plus specialist palliative care--creating a more sustainable model. N Engl J Med 2013;368:1173-5. doi:10.1056/NEJMp1215620.

2 Krakauer EL, Rajagopal MR. End-of-life care across the world: a global moral failing. Lancet 2016;388:444-6. doi:10.1016/ S0140-6736(16)31133-3

3 Centeno C, Lynch T, Garralda E, Carrasco JM, Guillen-Grima F, Clark D. Coverage and development of specialist palliative care services across the World Health Organization European Region (2005-2012) Results from a European Association for Palliative Care Task Force survey of 53 Countries. Palliat Med 2016;30:351-62 doi:10.1177/0269216315598671

4 World Health Organization. WHO Definition of Palliative Care, http:// www.dgpalliativmedizin.de/images/stories/WHO_Definition_2002 Palliative_Care englisch-deutsch.pdf.

5 Smith TJ, Temin S, Alesi ER, et al. American Society of Clinical Oncology provisional clinical opinion: the integration of palliative care into standard oncology care. / Clin Oncol 2012;30:880-7. doi:10.1200/ JCO.2011.38.5161.

6 Ferrell BR, Temel JS, Temin S, et al. Integration of Palliative Care Into Standard Oncology Care: American Society of Clinical Oncology Clinical Practice Guideline Update. / Clin Oncol 2017:35:96-112. doi:10.1200/JCO.2016.70.1474.

7 Zimmermann C, Riechelmann R, Krzyzanowska M, Rodin G, Tannock I. Effectiveness of specialized palliative care: a systematic review. JAMA 2008;299:1698-709. doi:10.1001/jama.299.14.1698.

8 Rohani C, Abedi H-A, Omranipour R, Langius-Eklöf A. Health-related quality of life and the predictive role of sense of coherence, spirituality and religious coping in a sample of Iranian women with breast cancer: a prospective study with comparative design. Health Qual Life Outcomes 2015:13:40 doi:10.1186/s12955-015-0229-1.

9 O'Connell KA, Skevington SM. The relevance of spirituality, religion and personal beliefs to health-related quality of life: themes from focus groups in Britain. BrJ Health Psychol 2005;10:379-98. doi:10.1348/135910705X25471.

10 Gaertner J, Siemens W, Antes G, et al. Specialist palliative care services for adults with advanced, incurable illness in hospital, hospice, or community settings--protocol for a systematic review. Syst Rev 2015:4:123. doi:10.1186/s13643-015-0121-4.

11 Moher D, Liberati A, Tetzlaff J, Altman DG. PRISMA Group. Preferred reporting items for systematic reviews and meta-analyses: the PRISMA statement. PLoS Med 2009;6:e1000097. doi:10.1371/journal. pmed.1000097.

12 Gaertner J, Siemens W, Daveson BA, et al. Of apples and oranges: Lessons learned from the preparation of research protocols for systematic reviews exploring the effectiveness of Specialist Palliative Care. BMC Palliat Care 2016;15:43. doi:10.1186/s12904-016-0110-y.

13 Coalition to Transform Advanced Care. The Coalition to Transform Advanced Care (C-TAC), http://www.thectac org/wp-content/ uploads/2015/02/C_TAC-Policy-Agenda.pdf.

14 Lefebvre C, Manheimer E, Glanville J. Searching for studies. In: Higgins JPT, Green S, eds. Cochrane Handbook for Systematic Reviews of Interventions. Cochrane Collaboration and Wiley \& Sons, 2008: 95-150doi:10.1002/9780470712184.ch6.

15 BMJ Clinical Evidence. Study design search filters, http:// clinicalevidence.bmi.com/x/set/static/ebm/learn/665076.html.
16 Bruera E, Higginson I, von Gunten CF. Textbook of Palliative Medicine. Taylor \& Francis, 2009

17 Ferrell BR, Coyle N. Oxford Textbook of Palliative Nursing. Oxford University Press, 2010 doi:10.1093/med/9780195391343. 001.0001.

18 Hanks G, Cherny NI, Christakis NA, Fallon M. Kaasa S, Portenoy RK. Oxford Textbook of Palliative Medicine. Oxford University Press, 2009doi:10.1093/med/9780198570295.001.0001.

19 Higgins JPT, Green S, eds. Cochrane Handbook for Systematic Reviews of Interventions. Cochrane Collaboration and Wiley \& Sons, 2008doi:10.1002/9780470712184.

20 Schünemann H, Brozek J, Guyatt G, Oxman A. GRADE Handbook: Introduction to GRADE Handbook, http://gdt.guidelinedevelopment. org/central_prod/_design/client/handbook/handbook.html.

21 Schünemann HJ, Oxman AD, Vist GE, et al. Interpreting results and drawing conclusions. In: Higgins JPT, Green S, eds. Cochrane Handbook for Systematic Reviews of Interventions. Cochrane Collaboration and Wiley \& Sons, 2008: 35988doi:10.1002/9780470712184.ch12.

22 Schwarzer G, Carpenter IR, Rücker G. Meta-Analysis with R.Springer, 2015doi:10.1007/978-3-319-21416-0.

23 Borenstein M, Hedges LV, Higgins JPT, Rothstein HR. Introduction to Meta-analysis.John Wiley \& Sons, 2009doi:10.1002/9780470743386.

24 Scott NW, Fayers PM, Aaronson NK, et al. EORTC QLQ-C30 Reference Values, http://groups.eortc.be/qol/manuals.

25 Osoba D, Rodrigues G, Myles J, Zee B, Pater J. Interpreting the significance of changes in health-related quality-of-life scores. I Clin Oncol 1998;16:139-44. doi:10.1200/JC0.1998.16.1.139.

26 Parmar MK, Torri V, Stewart L. Extracting summary statistics to perform meta-analyses of the published literature for survival endpoints. Stat Med 1998:17:2815-34 doi:10.1002/ (SICI)1097-0258(19981230)17:24<2815::AID-SIM110>3.0.CO;2-8.

27 Veroniki AA, Jackson D, Viechtbauer W, et al. Methods to estimate the between-study variance and its uncertainty in meta-analysis. Res Synth Methods 2016;7:55-79. doi:10.1002/jrsm.1164.

28 Wallen GR, Baker K, Stolar M, et al. Palliative care outcomes in surgical oncology patients with advanced malignancies: a mixed methods approach. Qual Life Res 2012;21:405-15. doi:10.1007/ s11136-011-0065-7.

29 Cheung W, Aggarwal G, Fugaccia E, et al. Palliative care teams in the intensive care unit: a randomised, controlled, feasibility study. Crit Care Resusc 2010;12:28-35

30 Gade G, Venohr I, Conner D, et al. Impact of an inpatient palliative care team: a randomized control trial. J Palliat Med 2008;11:180-90. doi:10.1089/jpm.2007.0055.

31 Hanks GW, Robbins M, Sharp D, et al. The imPaCT study: a randomised controlled trial to evaluate a hospital palliative care team. Br / Cancer 2002:87:733-9. doi:10.1038/sj.bjc.6600522.

32 Rabow MW, Dibble SL, Pantilat SZ, McPhee SJ. The comprehensive care team: a controlled trial of outpatient palliative medicine consultation. Arch Intern Med 2004:164:83-91. doi:10.1001/ archinte.164.1.83.

33 Zimmermann C, Swami N, Krzyzanowska M, et al. Early palliative care for patients with advanced cancer: a cluster-randomised controlled trial. Lancet 2014:383:1721-30. doi:10.1016/ S0140-6736(13)62416-2.

34 Jordhøy MS, Fayers P, Loge JH, Ahlner-Elmqvist M, Kaasa S. Quality of life in palliative cancer care: results from a cluster randomized trial. J Clin Oncol 2001;19:3884-94. doi:10.1200/JC0.2001.19.18.3884.

35 Jordhøy MS, Fayers P, Saltnes T, Ahlner-Elmqvist M, Jannert M, Kaasa S. A palliative-care intervention and death at home: a cluster randomised trial. Lancet 2000;356:888-93. doi:10.1016 S0140-6736(00)02678-7.

36 Sidebottom AC, Jorgenson A, Richards H, Kirven J, Sillah A. Inpatient palliative care for patients with acute heart failure. outcomes from a randomized trial. J Palliat Med 2015;18:134-42. doi:10.1089/ jpm.2014.0192.

37 Grudzen CR, Richardson LD, Johnson PN, et al. Emergency Department-Initiated Palliative Care in Advanced Cancer: A Randomized Clinical Trial. JAMA Oncol 2016. doi:10.1001/ jamaoncol.2015.5252.

38 Temel JS, Greer JA, Muzikansky A, et al. Early palliative care for patients with metastatic non-small-cell lung cancer. N Engl J Med 2010;363:733-42. doi:10.1056/NEJMoa1000678.

39 Cella DF Tulsky DS, Gray G, et al The Functional Assessment of Cance Therapy scale: development and validation of the general measure. / Clin Oncol 1993;11:570-9. doi:10.1200/JCO.1993.11.3.570

40 Cella DF, Bonomi AE, Lloyd SR, Tulsky DS, Kaplan E, Bonomi P. Reliability and validity of the Functional Assessment of Cancer Therapy-Lung (FACT-L) quality of life instrument. Lung Cancer 1995;12:199-220. doi:10.1016/0169-5002(95)00450-F.

41 Peterman AH, Fitchett G, Brady MJ, Hernandez L, Cella D. Measuring spiritual well-being in people with cancer: the functional assessment of chronic illness therapy--Spiritual Well-being Scale (FACIT-Sp). Ann Behav Med 2002:24:49-58, doi:10.1207/ S15324796ABM2401_06. 
42 Middel B, Bouma J, de Jongste $M$, et al. Psychometric properties of the Minnesota Living with Heart Failure Questionnaire (MLHF-Q). Clin Rehabil 2001:15:489-500 doi:10.1191/026921501680425216.

43 Supportive Care of the Dying. A Coalition for Compassionate Care. Supportive Care of the Dying: Modified City of Hope Patient Questionnaire and CALL Care Cover Letters, http://www. promotingexcellence.org/tools/pe6020.html.

44 Padilla GV. Quality of Life Cancer Scale (QOL-CA), https://eprovide. mapi-trust.org/instruments/quality-of-life-cancer-scale.

45 Greer JA, Tramontano AC, McMahon PM, et al. Cost Analysis of a Randomized Trial of Early Palliative Care in Patients with Metastatic Nonsmall-Cell Lung Cancer. J Palliat Med 2016;19:842-8. doi:10.1089/ jpm.2015.0476.

46 Lachin JM. Fallacies of last observation carried forward analyses. Clin Trials 2016;13:161-8. doi:10.1177/1740774515602688.

47 Committee for Medicinal Products for Human Use (CHMP). Guideline on Missing Data in Confirmatory Clinical Trials. EMA/CPMP/ EWP/1776/99 Rev 1, http://www.ema.europa.eu/ema/.

48 Mcllvennan CK, Allen LA. Palliative care in patients with heart failure. BMJ 2016:353:i1010. doi:10.1136/bmj.i1010.

49 Gaertner J, Maier B-O, Radbruch L. Resource allocation issues concerning early palliative care. Ann Palliat Med 2015;4:156-61.
50 Basch E, Deal AM, Kris MG, et al. Symptom Monitoring With Patient-Reported Outcomes During Routine Cancer Treatment: A Randomized Controlled Trial. J Clin Oncol 2016;34:557-65. doi:10.1200/JCO.2015.63.0830

51 Glare PA, Semple D, Stabler SM, Saltz LB. Palliative care in the outpatient oncology setting: evaluation of a practical set of referral criteria. J Oncol Pract 2011;7:366-70. doi:10.1200/JOP.2011.000367.

52 Maltoni M, Scarpi E, Dall'Agata M, et al. Early Palliative Care Italian Study Group (EPCISG). Systematic versus on-demand early palliative care: results from a multicentre, randomised clinical trial. Eur / Cancer 2016;65:61-8. doi:10.1016/j.ejca.2016.06.007.

53 Bakitas MA, Tosteson TD, Li Z, et al. Early Versus Delayed Initiation of Concurrent Palliative Oncology Care: Patient Outcomes in the ENABLE III Randomized Controlled Trial. J Clin Oncol 2015;33:1438-45. doi:10.1200/JCO.2014.58.6362.

54 Shih W. Problems in dealing with missing data and informative censoring in clinical trials. Curr Control Trials Cardiovasc Med 2002;3:4. doi:10.1186/1468-6708-3-4.

55 Freedman B. Equipoise and the ethics of clinical research. N Engl J Med 1987:317:141-5. doi:10.1056/NEIM198707163170304.

56 Campbell M, Fitzpatrick R, Haines A, et al. Framework for design and evaluation of complex interventions to improve health. $B M$ J 2000;321:694-6. doi:10.1136/bmi.321.7262.694.

Appendix: Supplementary text, tables and figures 